\title{
Update on indications, complications, and outcomes of scleral contact lenses
}

\author{
Raul E. Ruiz-Lozano ${ }^{1}$, Daniela E. Gomez-Elizondo ${ }^{1}$, Maria F. Colorado-Zavala ${ }^{1}$, \\ Denise Loya-Garcia ${ }^{1}$ and Alejandro Rodriguez-Garcia ${ }^{1}$ \\ ${ }^{1}$ Tecnologico de Monterrey, School of Medicine and Health Sciences, Institute of Ophthalmology and Visual Sciences, Monterrey, \\ Mexico
}

\begin{abstract}
Background: The role of scleral contact lenses (SCLs) has increasingly expanded since the first lens was fitted more than a century ago. While it was initially prescribed for the management of severely compromised corneas, the indications for modern SCL use have expanded to include less severe diseases. In this review, we aimed to provide an up-to-date overview of the current indications, complications, and outcomes for the various types of SCLs.

Methods: In this narrative review, we thoroughly searched the PubMed/MEDLINE database for literature published from January 1980 to November 2021. Only relevant up-to-date English references were included. Furthermore, the figures in this manuscript were derived from our unit's patient documentation.

Results: Currently, SCLs can successfully be used to manage ocular surface diseases, visually rehabilitate irregular corneas, and correct irregular refractive errors. Although newer materials have yielded the same visual outcomes with fewer complications, these consequences still occur in approximately one-third of contact lens wearers, including difficulties in insertion and/or removal, discomfort or pain, and developing either halos, blurriness, or haze. Even though most of these complications are minor and can be easily treated, a good practice is essential to avoid sight-threatening complications such as microbial keratitis.

Conclusions: SCLs are indispensable in ophthalmic clinics. The development of better-quality SCLs has increased the number of indications and improved the achievable visual rehabilitation. The future of developing improvements in SCL design, materials, and fit, and the expansion of their indication range is promising.
\end{abstract}

\section{KEYWORDS}

scleral contact lens, prosthetic replacement of the ocular surface environment, PROSE, corneal ectasia, irregular astigmatism, ocular surface disease, patient education, microbial keratitis

\section{INTRODUCTION}

Scleral contactlenses (SCLs) are wide-diameter, rigid, gas-permeable systems that vault over the whole cornea, creating a fluid reservoir between the anterior surface of cornea and posterior surfaces of the lens. The use of SCLs dates back to the 19th century, when the first SCLs made of blown glass, followed by those made of polymethylmethacrylate (PMMA), were designed. Their lack of oxygen permeability, however, limited widespread use [1].

Correspondence: Alejandro Rodriguez-Garcia, Clinical Professor of Ophthalmology, Avenue Batallon de San Patricio 112 Piso 1 Ote., Colonia Real de San Agustin. San Pedro Garza Garcia, Nuevo Leon, Mexico. CP 66278. Telephone No. +52 (81) 8888-0551. Fax No. +52 (81) 8356-1799. Email: immuneye@gmail. com. ORCID iD: https://orcid.org/0000-0002-1419-2109

How to cite this article: Ruiz-Lozano RE, Gomez-Elizondo DE, Colorado-Zavala MF, Loya-Garcia D, Rodriguez-Garcia A. Update on indications, complications, and outcomes of scleral contact lenses. Med Hypothesis Discov Innov Ophthalmol. 2021 Winter; 10(4): 165-178. https://doi.org/10.51329/mehdiophthal 1435

Received: 15 August 2021; Accepted: 23 November 2021

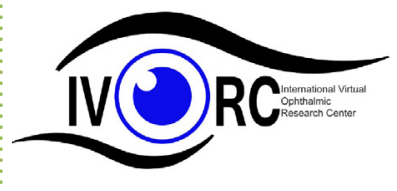

Copyright (C) Author(s). This is an open-access article distributed under the terms of the Creative Commons Attribution-NonCommercial 4.0 International License (http://creativecommons.org/licenses/by-nc/4.0/) which permits copy and redistribute the material just in noncommercial usages, provided the original work is properly cited. (c) (i) (s) 
Initially, the correction of refractive errors, mainly that of irregular astigmatism, was the primary indication for SCLs. Modern SCL designs and their unique fitting characteristics provide the ocular surface with continuous hydration and protection from the shearing forces of the eyelids [2]. Nowadays, reasons for SCLs prescription are myriad, including visual rehabilitation in patients with corneal irregularities (i.e., scarring, ectasia), ocular surface diseases (OSDs; i.e., neurotrophic keratopathy $[\mathrm{NK}]$, cicatrizing conjunctivitis), aphakic and nonaphakic refractive errors, and cosmesis [3-5].

Even though SCL use is generally safe, ophthalmologists and optometrists must remain cognizant of SCLrelated complications that may be sight-threatening. Patient education regarding the correct use of SCLs remains the most important measure to prevent complications. The purpose of this review was to provide an up-to-date overview of the current indications, complications, and outcomes for and of the use of the various types of SCLs.

\section{METHODS}

In this narrative review, we thoroughly searched the PubMed/MEDLINE database for literature using the following keywords: "scleral lens," "prosthetic replacement of the ocular surface environment (PROSE)," "corneal ectasia," "irregular astigmatism," and "ocular surface disease". We reviewed papers published between January 1980 and November 2021, focusing on studies published after 2000. Only English references were included. Furthermore, the figures in this manuscript were derived from our unit's patient documentation. Prior to including figures in this review, we obtained informed consent from the patients.

\section{RESULTS}

The recent surge in interest in SCLs has resulted in a muddled understanding of the terminology used to describe numerous emerging lens designs. Moreover, the naming schemes developed by many SCL manufacturers and distributors has increased such confusion [4]. The Scleral Lens Education Society (SLS) proposed an internationally recognized naming system to characterize SCLs based on fit and size features [4, 5]. According to the SLS, the most relevant aspect is where the lens rests: either the cornea, sclera, or both. Regarding size, the SLS classifies SCLs as semi-scleral (12.5-15 mm), mini-scleral (15-18 mm), and large scleral (18-25 mm) [5].

SLS developed standardized terminology using scientific language to cover all elements of SCL fabrication, fitting, and management [6]. Table 1 shows the summarized lens parameters with their respective acronyms and measurement units $[6,7]$. This new terminology allows manufacturers to provide uniform labeling information to ophthalmologists and optometrists, facilitate customized lens fitting, and compare various designs.

The SCL design is characterized by the made material, diameter, base curve radius, optic zone, peripheral curvature, and other specific features. Table 2 shows the specifics of SCL designs, manufacturers, and indications according to the largest series reported [2, 8-14].

\section{DISCUSSION}

The main indications for SCLs are visual rehabilitation in patients with corneal irregularities; the treatment of OSDs and dry eye; correction of refractive errors, including keratoconus $(\mathrm{KCN})$ and irregular astigmatism; cosmesis; and the prevention of sport injuries due to sports practice [1,2,5]. According to the Scleral Lenses in Current Ophthalmic Practice Evaluation (SCOPE), in 2015, 74\% of SCLs were recommended for corneal irregularity, $16 \%$ for OSDs, and $10 \%$ for refractive errors [15].

\section{Visual rehabilitation in patients with corneal irregularity}

Primary corneal ectasias (PCE) manifest as progressive eccentric thinning and steepening of the cornea, which induces irregular astigmatism [16]. KCN, pellucid marginal degeneration (PMD), and keratoglobus (KG) represent the specific patterns of PCE [14]. KCN is the most common PCE and the most frequent indication for SCL fitting [15] (Figure 1A). A study including 89 eyes with corneal ectasia (mean steep keratometry reading of

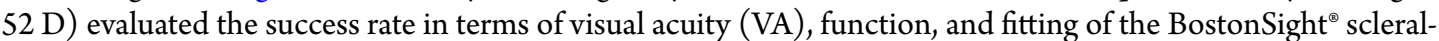
fitted lens (Needham, MA, USA) [14]. In all of the eyes, the BostonSight ${ }^{\circledR}$ SCL was successfully fitted. Significant improvement of Snellen VA (from 20/70 to 20/25), and visual functioning measured by the 25-item National Eye Institute Visual Function Questionnaire (NEI VFQ-25) (27.6 points improvement) were also noted [14]. Similar results were obtained by DeLoss et al. after comparing 36 keratoconic eyes fitted with the BostonSight ${ }^{\circledR}$ SCL with 37 eyes that underwent keratoplasty [17]. Eyes fitted with the BostonSight ${ }^{\oplus}$ SCL achieved a better VA and faster visual improvement [17]. Even in patients with $\mathrm{KCN}$ who developed acute corneal hydrops [18], 
Table 1. Scleral contact lens parameters

\begin{tabular}{|c|c|}
\hline Lens parameters & Definition \\
\hline Power & Back vertex optical power of the scleral contact lens. \\
\hline Primary functional lens diameter & $\begin{array}{l}\text { Scleral contact lens diameter, and characterized by a chord of where the contact } \\
\text { lens first touches or "lands" on the ocular surface along a similar meridian. }\end{array}$ \\
\hline Primary functional sagittal depth of the lens & $\begin{array}{l}\text { Distance from the apex of the back surface of the scleral contact lens } \\
\text { perpendicular at a chord characterized by the PFLD; is a theoretical value of } \\
\text { where the contact lens would contact the conjunctiva. }\end{array}$ \\
\hline Back Optic Zone Radius & $\begin{array}{l}\text { Distance from a centerline or point to an axis of rotation characterizing the } \\
\text { contact lens back optic zone; the central portion radius of curvature of the } \\
\text { contact lens posterior surface. }\end{array}$ \\
\hline Back Optic Zone Diameter & $\begin{array}{l}\text { In a scleral contact lens, the diameter of its back optic zone radius, which is } \\
\text { measured until the surrounding junction. }\end{array}$ \\
\hline Optical Zone Diameter & Diameter of central scleral contact lens zone that contains the optics. \\
\hline Transition Zone Radius and width & $\begin{array}{l}\text { The TZR is situated exactly peripheral to the optical zone, continuing to the } \\
\text { start of the landing zone. Its name is derived from its nature of transitioning } \\
\text { between the optic and landing zones. }\end{array}$ \\
\hline Landing Zone Radius and width & $\begin{array}{l}\text { Area peripheral to the TZR, starting at the primary functional diameter and } \\
\text { ending at the lens edge. This portion of the scleral contact lens aligns with } \\
\text { the ocular surface and the scleral conjunctiva and holds part of the entire lens } \\
\text { weight; the rest is supported with the fluid reservoir. }\end{array}$ \\
\hline Toric Peripheral Curves & System of peripheral curves that vary in radii among quadrants. \\
\hline Maximal lens thickness & $\begin{array}{l}\text { The thickest part of a scleral contact lens, with a notation concerning its precise } \\
\text { location. }\end{array}$ \\
\hline Oxygen transmissibility of the system $(\mathrm{Dk} / \mathrm{t})$ & $\begin{array}{l}\text { Calculated via dividing the Dk of the material by the lens thickness (in } \mathrm{cm} \text { ) of } \\
\text { the measured sample in specified conditions. }\end{array}$ \\
\hline Edge profile & $\begin{array}{l}\text { The shape of the edge in a plane comprising the scleral contact lens axis is } \\
\text { defined as standard if not modified from the manufacturer's original design } \\
\text { (modification should be specified in } \mu \mathrm{m} \text { ). }\end{array}$ \\
\hline Maximal Edge thickness & $\begin{array}{l}\text { Rating the thickness of a scleral contact lens measured normal to the front } \\
\text { surface, measured at the edge of the lens. }\end{array}$ \\
\hline Wetting angle of the material & $\begin{array}{l}\text { Measure of how wettable a given surface is. The steeper the angle, the greater } \\
\text { the decrease in the wettability of the lens surface [7]. }\end{array}$ \\
\hline Oblateness & $\begin{array}{l}\text { A lens design wherein the back optic zone radius is flatter than the peripheral } \\
\text { optic zone radius. }\end{array}$ \\
\hline Localized vaulting & $\begin{array}{l}\text { Disparity in one of the scleral contact lens zones to vault a specific area with } \\
\text { dissimilar values in } \mu \mathrm{m} \text {. }\end{array}$ \\
\hline Venting channels & $\begin{array}{l}\text { Modification to lens design of the LZR; used in patients where scleral contact } \\
\text { lenses seal off and impose suction to the eye, whereby incorporating VC can } \\
\text { relieve discomfort and enhance tear exchange. }\end{array}$ \\
\hline Notching & $\begin{array}{l}\text { Local variation in the scleral landing zone generates asymmetrical profiles to } \\
\text { accommodate the shape of a specific surface irregularity. }\end{array}$ \\
\hline Stabilization Process & $\begin{array}{l}\text { Stabilization is attained with several design features and should be announced } \\
\text { by its technology: PB, DSO, APCS, or TR. }\end{array}$ \\
\hline
\end{tabular}




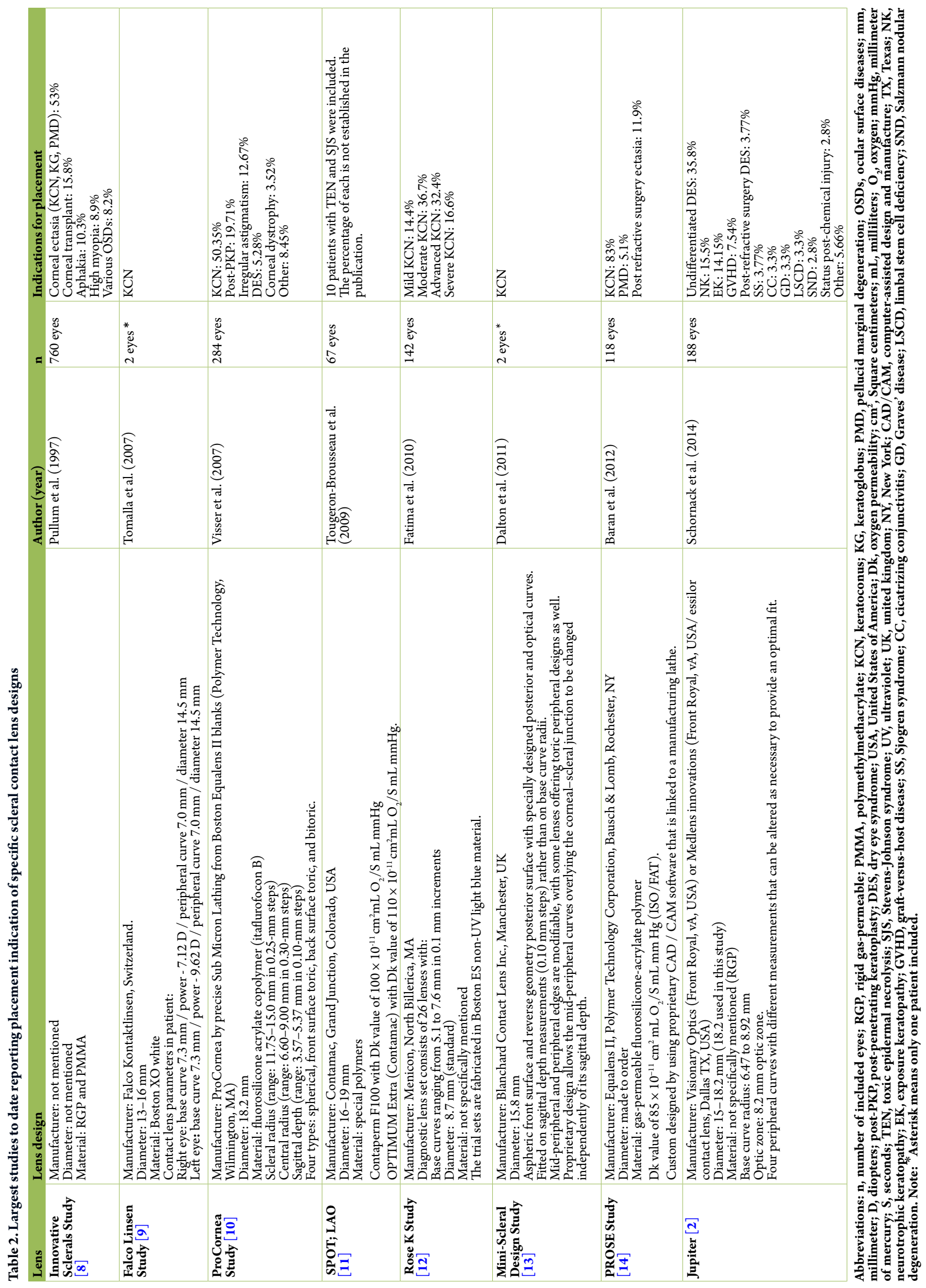




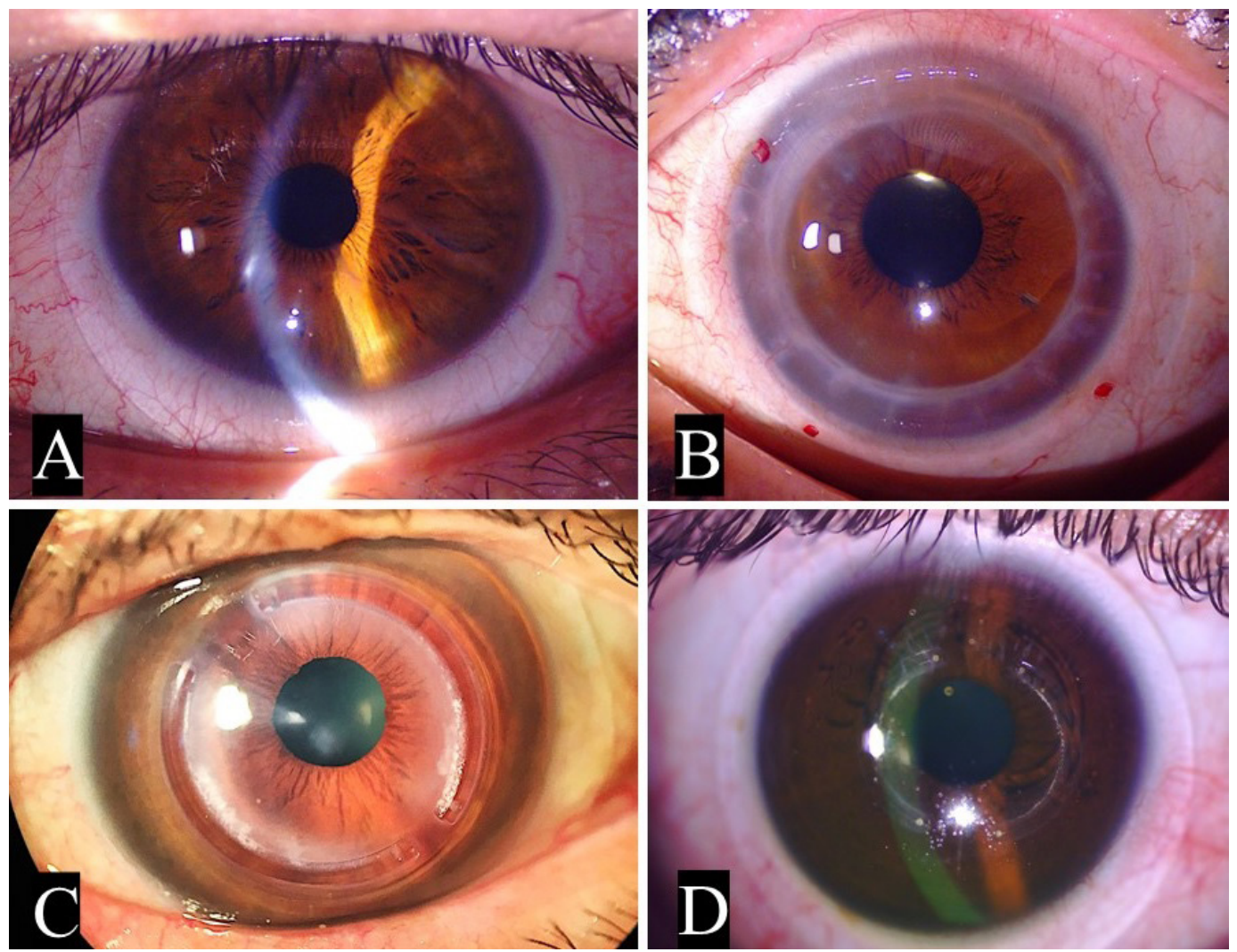

Figure 1. (A) Scleral contact lens (SCL) successful adaptation in a patient with advanced keratoconus with central corneal scarring due to previous gas permeable contact lens (GP-CL) wear. (B) Toric SCL adaptation process in a patient with high secondary astigmatism after corneal transplantation for keratoconus and high myopia. (C) Successful SCL adaptation in a patient with INTACS ${ }^{\circ}$ intracorneal ring segments (ICRS) insertion after laser in situ keratomileusis (LASIK)-induced keratectasia. (D) Keratoconic eye fitted with a scleral contact lens for final visual rehabilitation after Kerarings ${ }^{\circledR}$ ICRS insertion for corneal remodeling.

the mini-Misa SCL (Microlens, Arnhem, The Netherlands) was effective in providing good VA [19]. In a case series of $20 \mathrm{PMD}$ eyes fitted with the BostonSight ${ }^{\circledR} \mathrm{SCL}$, a substantial improvement in VA after 7.25 months was reported [20]. In patients with KG, Rathi et al. fitted two cases with the BostonSight ${ }^{\otimes}$ SCL. One eye developed corneal hydrops with deterioration of VA, while the other, with healed hydrops prior to lens wear, had successful VA improvement over a three-year follow-up [21].

Post-penetrating keratoplasty (post-PK) may result in significant irregular astigmatism leading to anisometropia, uncorrectable refractive errors with spectacles, and poor vision due to reduced contrast sensitivity [22] (Figure 1B). Historically, spherical rigid gas-permeable (RGP) lenses have been utilized for visual recovery in patients post-PK. Nevertheless, the abnormal corneal curvature results in several nuisances, including fitting difficulties, frequent decentration, contact lens (CL) ejection, dislocation risk, and significant intolerance [23]. Moreover, the interaction between the mechanical elements (the rigid lens and the cornea) may result in corneal microtrauma and epithelial and stromal disruption with subsequent ocular inflammation and an increased risk of graft rejection and infection [24]. Severinsky et al. evaluated the success rate, defined as tolerating the lenses for at least 10 hours per day, of SCLs for visual rehabilitation after PK in 28 patients (33 eyes) [24]. The mean follow-up time was 5.2 years (range $0.5-8.8$ ). Twenty-one patients (75\%) successfully wore SCLs for at least 10 $\mathrm{h}$ per day. Five patients (18\%) achieved partial success, which was defined as using the lenses between 6 and $10 \mathrm{~h}$ a day [24]. Similar results were achieved by Barnett et al. in 48 post-PK eyes using the Jupiter SCLs [23]. Thirtythree of these eyes $(69 \%)$ had previously worn other varieties of CLs, the most frequent of which was RGP. Compared with patients who ceased SCL use, patients who consistently used SCLs were more likely to report an excellent subjective vision. Almost all eyes $(n=44,91.7 \%)$ achieved a Snellen VA of 20/40 or better [23]. Irregular astigmatism and anisometropia often encountered in patients post-PK render SCLs a more suitable option than other CL types for visual rehabilitation. Because SCLs vault over the cornea with complete central and limbal clearance, it creates a better fit, less movement, less visual fluctuation, and better comfort [23, 24]. 

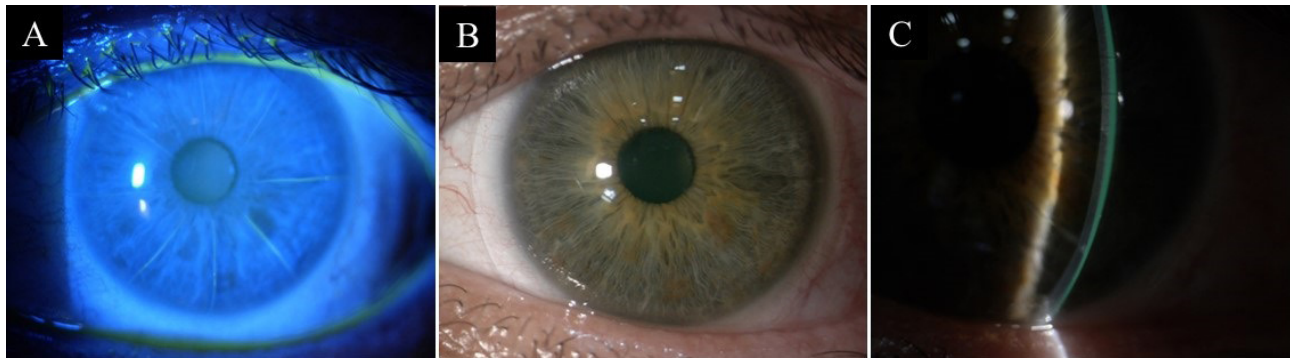

Figure 2. Slit-lamp photographs of a patient with irregular astigmatism after radial keratotomy. (A) Fluorescein-stained cornea demonstrating eight clean corneal radial incisions. (B) Scleral contact lens resting on the conjunctiva without vascular impingement. (C) Fluorescein-stained fluid reservoir vaulting the cornea.
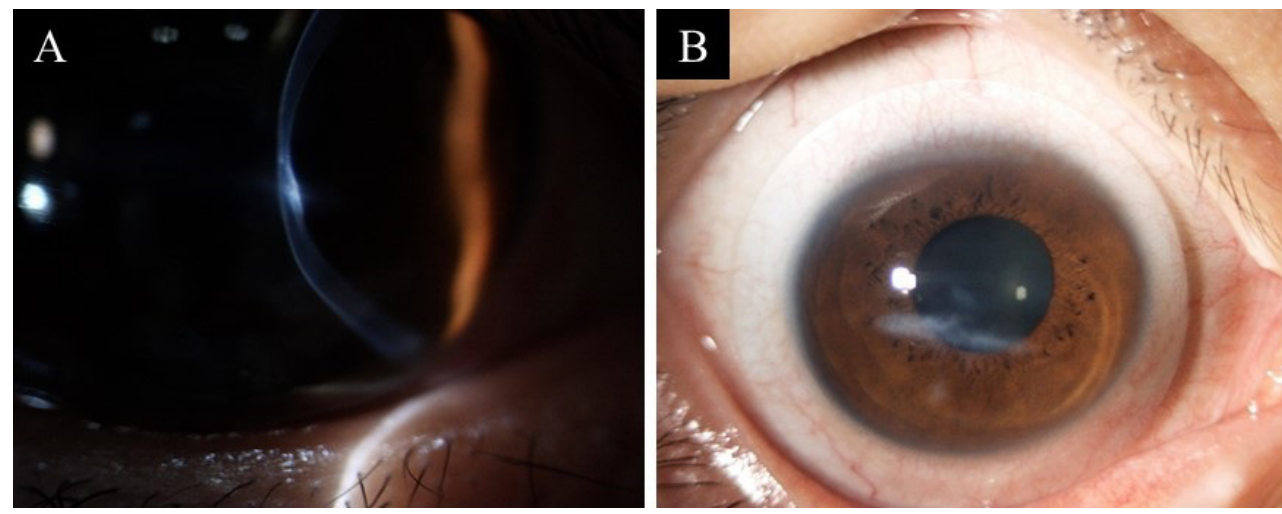

Figure 3. (A) Slit-lamp photographs of a patient with paracentral corneal thinning and scarring after corneal hydrops in advanced keratoconus. (B) Scleral contact lens vaulting the cornea from limbus to limbus with adequate scleral landing.

Although corneal refractive surgery is generally safe, it may result in significant dry eye, corneal neuralgia, optical aberrations, and irregular astigmatism, which could progress to ectasia [3, 25] (Figures 1C-D). Many patients with radial keratotomy suffer from a hyperopic shift and high or irregular astigmatism that cannot be corrected with spectacles, soft, and even RGP CLs. In such cases, SCLs have become an excellent visual rehabilitation alternative [3] (Figures 2A-C). Mian et al. evaluated the performance of the BostonSight ${ }^{\circledR}$ SCL in 44 patients with laser-assisted in situ keratomileusis (LASIK)-related complications, including corneal ectasia (53\%), other optical aberrations (32\%), dry eye (9\%), and corneal neuralgia (7\%) [25]. Of these patients, $86 \%$ were previously intolerant to other types of CLs. Almost all of the eyes (84\%) achieved a significant VA improvement [25]. Montalt et al. found a marked improvement in VA, subjective comfort ratings, and usage time (mean $12.67 \mathrm{~h}$ per day) with the use of the BostonSight ${ }^{\circ} \mathrm{SCL}$ in patients with irregular corneas after LASIK [26]. The authors also demonstrated an improvement in the corneal resistance factor, a biomechanical parameter of the cornea which is usually reduced after refractive surgery [26].

Corneal scarring may occur after a myriad of causes, including herpetic keratitis, corneal trauma, previous corneal infections, chemical and thermal burns, NK, corneal hydrops (Figures 3A, B), cicatrizing keratoconjunctivitis, and autoimmune corneal ulceration [27-29]. Scarring results in a reduced quality of vision because of leukoma formation, high-order aberrations, and irregular astigmatism. In 57 eyes with corneal opacity fitted with the BostonSight ${ }^{\circledR}$ SCL, Parra et al. demonstrated a significant visual improvement of up to five Snellen lines [30]. Since corneal scarring usually results from infectious and/or inflammatory pathologies, providing detailed lens care instruction is crucial to avoid SCL-related sight-threatening complications in this group of high-risk patients [3].

\section{Ocular surface disease (OSD)}

The PROSE, a custom-made prosthetic device, is used to improve the hindered ocular surface functions in various OSDs. It is a large-diameter gas-permeable SCL that provides high oxygen permeability, positional stability on the eye, sustained corneal humidity, and protection against lid shearing blinking forces. Thus, it creates an adequate environment that augments corneal surface healing [31]. 
Stevens-Johnson syndrome $(\mathrm{SjS})$ and toxic epidermal necrolysis (TEN) are acute, hypersensitive, mucocutaneous, bullous reactions that result in significant blistering and desquamation of the skin and mucous membranes [32]. Since both diseases affect every component of the ocular surface, soft and RGP corneal CLs fail to provide adequate protection; thus, their use is disfavored. In the largest retrospective cohort to date, Papakostas et al. reported the visual outcomes of 167 eyes with SJS or TEN managed with the PROSE device [31]. After 16 months of follow-up, the visual function, measured with the NEI VFQ-25 questionnaire, significantly improved [31]. The PROSE device also reported good visual outcomes in pediatric patients with SJS or TEN [32].

Chronic graft-versus-host disease (cGVHD) is a complication arising from either allogeneic hematological stem cell transplantation (allo-HSCT) from human leukocyte antigen (HLA)-matched donors or bone marrow transplantation [33]. In cGVHD, donor $\mathrm{CD}^{+}$and $\mathrm{CD} 8^{+} \mathrm{T}$-cells recognize host cells as non-self entities. The incidence of this entity varies between $10 \%$ to $90 \%$ of patients undergoing allo-HSCT [33]. Ocular manifestations include keratoconjunctivitis sicca (KCS), the most prevalent manifestation, chronic blepharitis, and corneal epithelial defects [3]. Chronic ocular inflammation causes conjunctival and corneal fibrosis, culminating in scarring and squamous metaplasia of the ocular surface [34]. Although the management approach of cGVHD is multimodal, the use of SCLs has proven worthwhile. In a large cohort of 875 eyes, including 50 with cGVHD, Rosenthal and Croteau evaluated the visual outcomes after fitting with the PROSE device [35]. The primary indication in all of these eyes was severe dry eye, and in four eyes, there was an associated persistent epithelial defect (PED). VA and function improved in 49 (98\%) eyes, and the PED healed in 3 (75\%) eyes with extended overnight wear of the PROSE lens. One eye, however, did not heal because of the presence of a descemetocele [35]. In another study, Agranat et al. demonstrated that compared to other forms of OSD, patients with cGVHD were more prone to continue wearing the PROSE device after 5 years [36].

Exposure keratopathy $(\mathrm{EK})$ is a condition when the cornea dries because of incomplete or inadequate lid closure. If left untreated, it can lead to epithelial breakdown, stromal melt, ulceration, and perforation [37]. A retrospective study by Gervasio et al. compared the VA outcomes of the PROSE lenses versus standard treatments (i.e., artificial tears, ointment, temporary tarsorrhaphy) in 27 and 26 eyes with postsurgical lagophthalmos and EK, respectively [38]. Eyes managed with the PROSE device had a significantly improved VA at 1, 3, 6, and 12 months. Moreover, eyes requiring the PROSE device were more likely to have failed multiple treatments [38]. In another series, the use of PROSE significantly improved the visual function (the OSD index) and corneal staining in eyes with EK [39]. The overnight use of SCLs were also found to effectively manage PEDs, a condition enhanced by EK [40]. Re-epithelization occurred in $85 \%$ of the eyes, with most eyes $(70.6 \%)$ achieving healing in $<12$ days [40].

$\mathrm{NK}$ is an impairment of corneal nerves that results in reduced or absent sensations, leading to abnormal nourishment and damage to the corneal epithelium with a higher risk of ulceration, infection, stromal thinning, and perforation [29]. SCLs are effective in the management of NK in eyes previously treated with soft CLs [35]. However, the use of any CLs in neurotrophic corneas requires extreme caution [3]. Reduced corneal sensitivity results in a hindered or absent response to infection symptoms, mainly pain [41], thus increasing the risk of unwanted complications, such as microbial keratitis (MK). Some authors advocate using prophylactic antibiotic eye drops to prevent infection in patients wearing SCLs [41].

Cicatrizing conjunctivitis (CC) comprises a myriad of local and systemic diseases that lead to conjunctival scarring. A myriad of etiologies, including SJS / TEN [32], ocular mucous membrane pemphigoid (OMMP) [27], ocular lichen planus [28, 42], cGVHD [33], and limbal stem cell deficiency (LSCD) [43], have been associated with conjunctival scarring [44]. The effective management of CC usually requires immunosuppressive therapy to treat the underlying disease and CLs and ocular surface surgery to treat its associated complications [44]. Although research on SCLs to date is limited to small-scale case series and case reports, SCLs play a role in the ocular surface protection and visual rehabilitation of patients with CC [45]. The use of SCLs in LSCD deserves special mention. Since up to $15 \%$ of LSCD cases are associated with soft and rigid corneal CLs, the use of a large-diameter tear-exchangeable rigid SCL is required in eyes with LSCD. The International LSCD Working Group suggests that rigid SCL may be used before or after reconstructing the ocular surface in eyes with LSCD [46].

PEDs occur when there is an impaired trophic function of the cornea. If left untreated, PEDs lead to stromal melting, scarring, ulceration, and perforation [29]. Rosenthal et al. [47] first reported the use of SCLs for the management of PEDs. Most eyes achieved complete re-epithelization. Nevertheless, four eyes (29\%) developed MK [47]. In a study by Lim et al., with the use of the PROSE device and prophylactic antibiotic eyedrops in the reservoir, there was successful re-epithelization in $85 \%$ of the assessed eyes with no cases of infection [40].

Sjogren's syndrome (SS) is a chronic rheumatic disease characterized by a progressive exocrine gland lymphocytic infiltration that can affect multiple organs and systems [48, 49]. La Porta Weber et al. fitted the 
Esclera (Mediphacos Inc., Belo Horizonte, Brazil) lenses in 41 eyes with severe dry eye disease (DED); of those, $11 \mathrm{had}$ associated SS [50]. The Esclera lens is a non-fenestrated lens with a 16- to 18.2 -mm diameter, with a $\mathrm{Dk} / \mathrm{t}$ of 141 . Although the statistical analysis was not separated between eyes with and without SS, all of the eyes were reported to have significant improvement in symptoms and quality of life. Moreover, the tear osmolarity was significantly reduced [50]. Another study, however, reported a significant increase in the HLA-DR isotype inflammatory marker in eyes with DED related to SS after one year of SCL use [51].

Corneal dystrophies (CD) constitute a group of hereditary non-inflammatory diseases that may involve the cornea at any level. They are subclassified by the affected anatomic location [52]. Patients with CD typically experience pain and blurred vision due to recurrent corneal erosions and irregular astigmatism, respectively [53]. Thus, manufacturers aim for SCLs to both improve VA and reduce discomfort. Unfortunately, evidence to date regarding the use of SCLs in CD is scarce. In a large-scale study performed by Parra et al., significant visual improvement was achieved in 17 out of 825 eyes (2.1\%) with CD after PROSE device fitting [30].

\section{Refractive errors}

Aphakia, when unilateral, produces significant aniseikonia, thus limiting the use of spectacles for optical correction. SCLs, specifically the mini-scleral design (MSD), are a good option for managing traumatic aphakia with concomitant irregular astigmatism secondary to corneal scarring [54]. Alipur and Hosseini evaluated the visual outcomes of the MSD in the optical treatment of individuals with traumatic aphakia associated with iridocorneal injury. The VA significantly improved in all of the assessed eyes, and the average time spent
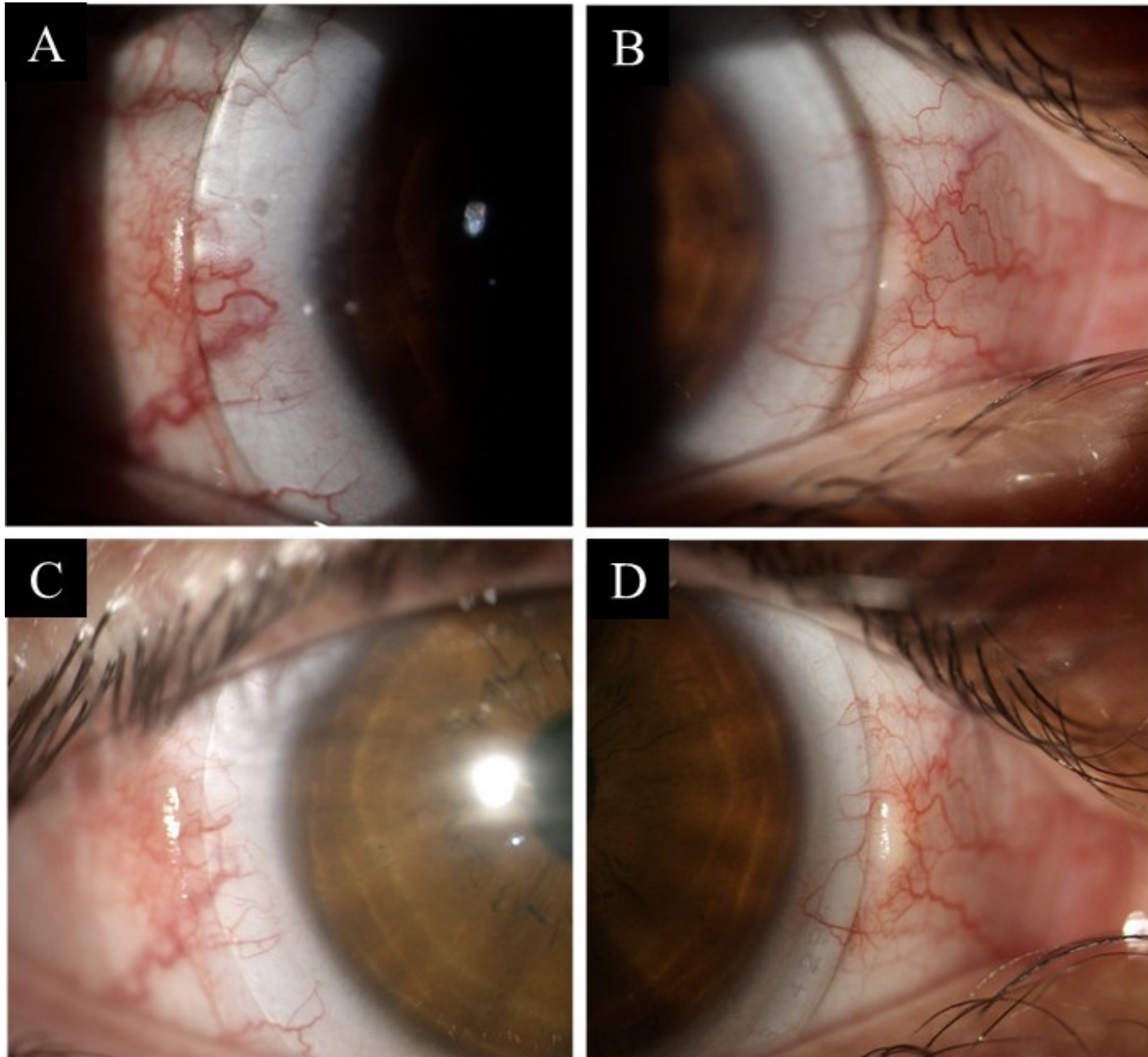

Figure 4. Slit-lamp photographs of a patient with scleral contact lens (SCL)-induced pingueculitis, presenting with focal conjunctival injection and irritation at the nasal $(A, C)$ and temporal $(B, D)$ pingueculae areas, compromising the SCL landing zone. 
comfortably wearing the MSD was $11.6 \mathrm{~h}$ (range 8-16 h). The only adverse effect observed was mild redness in two cases [54]. High ametropia (non-aphakic), as stated above, was first corrected with SCLs by August Müller in 1889 [55]. In 2005, Pullum et al. reported that high myopia was indicated in only $2.6 \%$ of the eyes $(21 / 808)$ that they examined [56]. Currently, the role of SCLs in the optical correction of high refractive error seems limited to either patients who cannot tolerate corneal CLs and/or have associated irregular astigmatism [3].

\section{Cosmetics, sports, and other indications}

Cosmetic tinted SCLs are made to enhance the appearance of a healthy eye by "modifying" the iris color. Even though these lenses are considered to be for esthetic purposes, misuse can lead to potentially blinding complications [3].

Therapeutic tinted lenses reduce glare and photophobia in patients with albinism, migraines, cone dystrophies, and retinitis pigmentosa. These lenses are typically red-tinted [3, 4]. At the time of writing, the role of tinted lenses to improve color perception in color-blind patients is controversial [57].

Prosthetic tinted lenses are prescribed to improve cosmesis in eyes with disfiguring diseases, including corneal scarring, bullous keratopathy, and microphthalmia [58]. However, current evidence is limited to soft CLs.

Ptosis may result from long-term, rigid corneal CL wear. Evidence obtained from case reports suggests that SCLs can effectively manage ptosis resulting from ocular myopathy, long-term use of RGP corneal CLs, myopathy due to Kearns-Sayre syndrome, and phthisis bulbi [59].

Sports practice, mainly those involving physical contact and those practicing underwater, is another frequent indication for SCL wear [4].

\section{Potential adverse events}

Non-severe adverse events: Despite the safety and efficacy profile of modern designs of SCLs, associated complaints are still frequent. Pecego et al. reported that $23 \%(25 / 107)$ of the eyes they investigated abandoned the Jupiter Scleral Lens after at least 3 months of wear [60]. At least 20\% complained of difficulties in insertion and/or removal, $10 \%$ experienced discomfort or pain, and 10\% developed either halos, blurriness, or haze [60]. In a clinical trial on eyes fitted with the Rose K2 XL semi-SCLs (Menicon Co. Ltd., Nagoya, Japan), during the follow-up period, Romero-Jiménez et al. noted corneal abrasions upon lens insertion and conjunctival injuries induced due to lens removal [61]. SCL-induced pingueculitis were also observed (Figures 4A-D). Tear debris accumulation behind the SCLs may result in reduced VA (Figure 5A-C). Rathi et al. reported a decrease in VA, ranging from one to seven lines in 14 eyes $(70 \%)$ after $4 \mathrm{~h}$ of wearing the Boston ${ }^{\circledast}$ Scleral Lens [62]. In all of
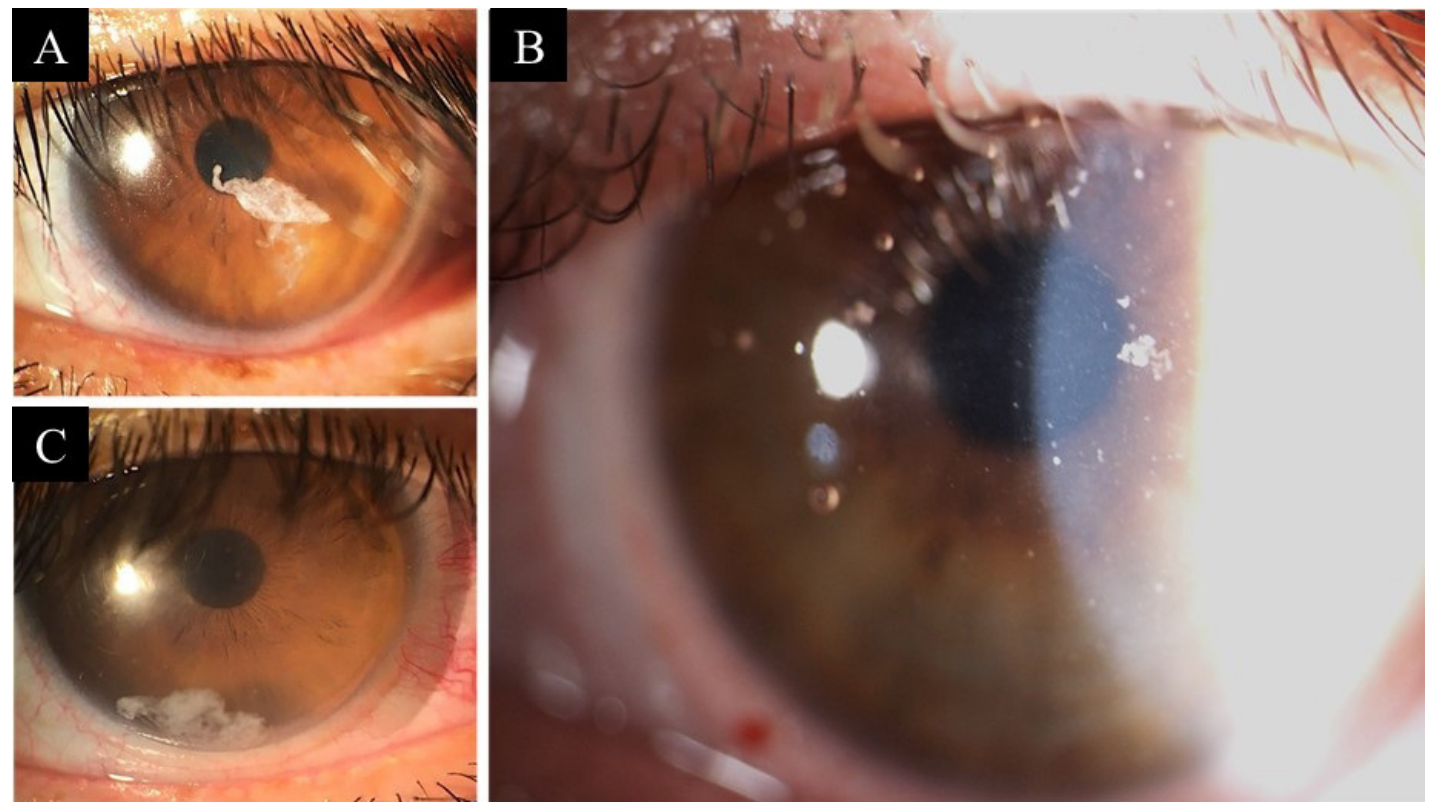

Figure 5. Slit-lamp photographs of patients with scleral contact lens fogging because of prominent tear debris accumulation (A-C) under the lens. 
the studied eyes, vision improved after removing and refilling the lens with new fluid [62]. An alternative to overcome this issue is removing and reinserting the lenses up to twice per day [4]. Hypoxia with SCL wear has significantly reduced with modern rigid SCLs. Vincent et al. reported a statistically significant but clinically insignificant corneal swelling of $<2 \%$ in healthy corneas after wearing the MSD lens for $8 \mathrm{~h}$ [63]. On the other hand, clinically significant corneal edema (across the central $6 \mathrm{~mm}$ ) was reported by Kumar et al. in nine postPKP eyes fitted with non-fenestrated SCLs with high Dk [64]. Lens refitting, hypertonic saline eye drops, and temporary lens discontinuation are strategies to manage clinically significant corneal edema [3]. Finally, CLassociated solution toxicity, which presents as mild punctate keratitis, is frequently reported among CL users [65] (Figures 6A, B). On the other hand, corneal infiltrative events (CIEs) are classified as symptomatic CL-induced peripheral ulcer, severe $\mathrm{MK}$, infiltrative keratitis, CL-induced red eye, asymptomatic infiltrative keratitis, and asymptomatic infiltrates [65]. Carnt et al. retrospectively analyzed the relationship between CIEs and solution toxicity in soft CL wearers. The authors concluded that eyes experiencing solution toxicity were more likely to experience CIEs (6.7\% that did versus $2.3 \%$ that did not experience toxicity) [65]. Overnight lens wear, reusable lens use, disinfection with multi-purpose solutions, lens case age $>6$ months, and rinsing with tap water are modifiable risk factors for CIE development. Meanwhile, age; a history of red eye; a high refractive error (>5 D); recent flu or colds; being a first-time lens wearer; and bacterial bioburden in the lid margin, bulbar conjunctiva, lens case, and/or in the CL itself, are non-modifiable risk factors [66].

Severe adverse events: Although rare, MK is the most feared complication associated with SCL wear. A recent review performed by Walker et al. reported 11 cases of MK among SCL wearers [67]. The most frequent indication for SCL fit was PED and post-PK irregular astigmatism in four and two eyes, respectively. EK (two eyes), OMMP and SS (one eye), GVHD (one eye), and NK (one eye) were the other indications for SCL placement. Staphylococcus epidermidis was the most frequent organism cultured in three eyes [67]. Risk factors included OSD, overnight wear, epithelial compromise, poor compliance, or steroid use [3]. Rosenthal and Croteau reported four cases of MK in patients with post-PK PED who wore SCLs overnight. In all of these eyes, the graft eventually failed. The authors suggested switching to a daily-wear schedule soon after PED resolution [35].

\section{Patient education}

As with other CL forms, thorough handwashing with a mild soap must be performed before any manipulation, insertion, or removal. The lens should be rinsed before being applied to the eye to remove the cleaning agent,

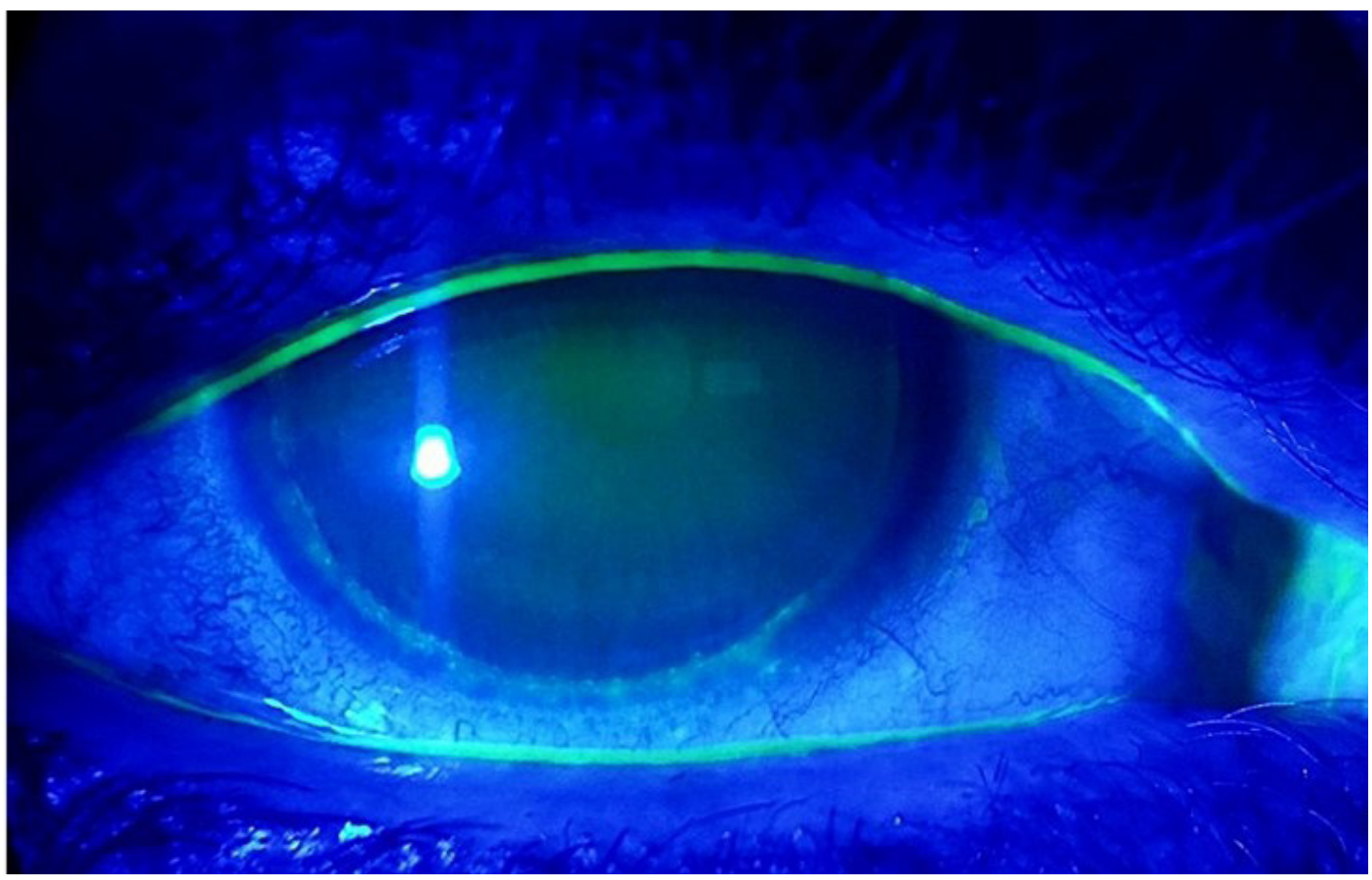

Figure 6. Slit-lamp photograph under diffuse illumination and fluorescein staining show diffuse moderate punctate corneal epithelial staining associated with contact lens solution toxicity in a scleral contact lens user. 
preventing irritation and chemical toxicity of the cornea [68]. Before insertion, users must fill the scleral reservoir with sterile non-preserved saline to avoid bubble formation [3]. In a previous study, bubbles trapped behind the lens were the most frequent complication during lens insertion [69]. A plunger (either ventilated or not) or a scleral cup may stabilize the lens, thus facilitating the insertion. A few commercially available plungers in ventilated and non-ventilated designs aid with SCL insertion and removal $[3,70]$.

There are two approaches for lens insertion. The first is the three-finger method, in which the patient uses their thumb, index, and middle finger to create a tripod stand for the SCL. Next, the lens is filled with sterile nonpreserved saline, and the eyelids are held open for insertion. The second method utilizes a large plunger; the lens must also be filled with solution. The lids are then opened with the non-dominant hand, and the patient leans forward parallel to the ground towards the lens until it touches the central cornea. The plunger must be cleaned with alcohol or peroxide following each use. The patient should examine the eye after lens insertion to look for air bubbles that can produce discomfort, decrease vision, and cause corneal desiccation with time [70].

Most patients rely on their lenses for functional vision and, therefore, use them during waking hours. Consultants in the SCOPE survey reported advising wearing SCLs for $12 \mathrm{~h}$ (range: $2-19 \mathrm{~h}$ ) [71]. In some cases, patients required overnight wear to avoid symptom worsening upon awakening [72]. Highly gas-permeable materials, such as hydrogel, allow overnight wear. In such cases, daily removal for cleaning and refilling with saline is advised [3].

SCLs should be cleaned by rubbing them for 15 seconds and then rinsing them with saline or multi-purpose solution before overnight storage. After the initial cleaning, lenses must be placed overnight in a disinfecting solution. Many multipurpose solutions are suitable for storing, such as those containing $3 \%$ hydrogen peroxide or chlorhexidine [68].

SCLs have a sealed fit, and any contamination can expose the eye to infection during the time of their wear. Therefore, strict compliance is crucial while using these lenses. Compliance can sometimes be complicated because of the complexity of the prescribed lens regimen and the underlying management of conditions [3]. Drops applied over an SCL will not have direct exposure to the cornea or the anterior chamber as they would over a hydrophilic soft CL; thus, patients should be instructed to remove the lenses for cleaning and disinfection prior to drop instillation [3].

The follow-up schedule will depend on the underlying condition for which the SCL is prescribed. A retrospective study of 6,385 eyes of 6,188 patients by Zhu et al. recommended weekly follow-ups [73]. However, other conditions, such as filamentary keratitis in patients with adenoviral keratoconjunctivitis, can be monitored more closely. Moreover, close follow-ups and patient education are of utmost importance, especially in the case of overnight users [3].

The limitations of our review were related to the paucity of randomized control trials that evaluated SCL tolerance, safety, adverse events, wearing time, and complication rates. Moreover, current evidence on SCLs as a management for NK and several relatively rare diseases, such as SJS / TEN, is limited to a few case reports and case series. Thus, the information presented must be interpreted cautiously. The strengths of our review included presenting an up-to-date overview concerning the indications, complications, and outcomes for and of SCLs for almost every eye condition. Future randomized controlled studies are required to best evaluate the safety, efficacy, and outcomes of SCLs in every current indication for their use, including refractive errors and ocular surface pathology.

\section{CONCLUSIONS}

SCLs are an indispensable tool in ophthalmic and optometry practices, particularly in corneal, refractive, and OSD clinics. The technological development that lead to better SCLs has allowed a considerable increase in their clinical indications, resulting in a significant improvement in the resolution of severe OSDs, including severe $\mathrm{DED}, \mathrm{EK}, \mathrm{NK}$, and PEDs. It has also significantly improved the visual rehabilitation of patients with irregularities of the anterior surface of the cornea, irregular astigmatism, and corneal ectasia, considerably reducing the number of corneal transplant surgeries. Potential complications of SCLs are related to a poor fit, patient misuse, risk factors favoring complications, and intolerance. Eye care specialists must educate patients on the appropriate use, risks of misuse, and complications of SCLs.

\section{ETHICAL DECLARATIONS}

Ethical approval: The figures in this manuscript were derived from our unit's patient documentation. We obtained informed consent from the patients prior to including figures in this review.

Conflict of interest: None. 


\section{FUNDING}

This research was partially funded by the Immuneye Foundation, Monterrey, Mexico.

\section{ACIKNOWLED GEMENTS}

\section{None.}

\section{REFERENCES}

1. Schornack MM. Scleral lenses: a literature review. Eye Contact Lens. 2015;41(1):3-11. doi: 10.1097/ICL.0000000000000083 pmid: 25536528

2. Schornack MM, Pyle J, Patel SV. Scleral lenses in the management of ocular surface disease. Ophthalmology. 2014;121(7):1398-405. doi: 10.1016/j.ophtha. 2014.01.028pmid: 24630687

3. Jacobs DS, Carrasquillo KG, Cottrell PD, Fernandez-Velazquez FJ, Gil-Cazorla R, Jalbert I, et al. CLEAR - Medical use of contact lenses. Cont Lens Anterior Eye. 2021;44(2):289-329. doi: 10.1016/j.clae. 2021.02.002pmid: 33775381

4. van der Worp E, Bornman D, Ferreira DL, Faria-Ribeiro M, Garcia-Porta N, Gonzalez-Meijome JM. Modern scleral contact lenses: A review. Cont Lens Anterior Eye. 2014;37(4):240-50. doi: 10.1016/j.clae. 2014.02.002pmid: 24631015

5. Harthan JS, Shorter E. Therapeutic uses of scleral contact lenses for ocular surface disease: patient selection and special considerations Clin Optom (Auckl). 2018;10:65-74. doi: 10.2147/OPTO.S 144357pmid: 30319297

6. Michaud L, Lipson M, Kramer E, Walker M. The official guide to scleral lens terminology. Cont Lens Anterior Eye. 2020;43(6):52934. doi: 10.1016/j.clae.2019.09.006 pmid: 31561849

7. Shin HS, Jang JK, Kwon YS, Mah KC. Surface modification of rigid gas permeable contact lens treated by using a low-temperature plasma in air. Journal of the Korean Physical Society. 2009;55(6):2436-40. doi: 10.3938/jkps.55.2436

8. Pullum KW, Buckley RJ. A study of 530 patients referred for rigid gas permeable scleral contact lens assessment. Cornea. 1997;16(6):612-22 pmid: 9395869

9. Tomalla M, Cagnolati W. Modern treatment options for the therapy of keratoconus. Cont Lens Anterior Eye. 2007;30(1):61-6. doi: 10.1016/j.clae.2006.12.004 pmid: 17207654

10. Visser ES, Visser R, van Lier HJ, Otten HM. Modern scleral lenses part I: clinical features. Eye Contact Lens. 2007;33(1):13-20. doi: 01/10.1097.icl.0000233217.68379.d5 pmid: 17224674

11. Tougeron-Brousseau B, Delcampe A, Gueudry J, Vera L, Doan S, Hoang-Xuan T, et al. Vision-related function after scleral lens fitting in ocular complications of Stevens-Johnson syndrome and toxic epidermal necrolysis. Am J Ophthalmol. 2009;148(6):852-9 e2. doi 10.1016/j.ajo.2009.07.006 pmid: 19837383

12. Fatima T, Acharya MC, Mathur U, Barua P. Demographic profile and visual rehabilitation of patients with keratoconus attending contact lens clinic at a tertiary eye care centre. Cont Lens Anterior Eye. 2010;33(1):19-22. doi: 10.1016/j.clae.2009.09.004 pmid: 19939725

13. Dalton K, Sorbara L. Fitting an MSD (mini scleral design) rigid contact lens in advanced keratoconus with INTACS. Cont Lens Anterior Eye. 2011;34(6):274-81 doi: 10.1016/j.clae.2011.05.001 pmid: 21664856

14. Baran I, Bradley JA, Alipour F, Rosenthal P, Le HG, Jacobs DS. PROSE treatment of corneal ectasia. Cont Lens Anterior Eye. 2012;35(5):222-7. doi: 10.1016/j.clae.2012.04.003 pmid: 22633003

15. Nau CB, Harthan J, Shorter E, Barr J, Nau A, Chimato NT, et al. Demographic Characteristics and Prescribing Patterns of Scleral Lens Fitters: The SCOPE Study. Eye Contact Lens. 2018;44 Suppl 1:S265-S72. doi: 10.1097/ICL.00000000000000399 pmid: 28617729

16. Ruiz-Lozano RE, Garcia-de la Rosa G, Bustamante-Arias A, Davila-Cavazos O, Hernandez-Camarena JC. Corneal Hydrops Associated with Keratoconus in a Young Girl with Severe Allergic Conjunctivitis. J Allergy Clin Immunol Pract. 2021;9(3):1376-7. doi: 10.1016/j.jaip.2020.10.042 pmid: 33229290

17. DeLoss KS, Fatteh NH, Hood CT. Prosthetic Replacement of the Ocular Surface Ecosystem (PROSE) scleral device compared to keratoplasty for the treatment of corneal ectasia. Am J Ophthalmol. 2014;158(5):974-82. doi: 10.1016/j.ajo.2014.07.016 pmid: 25058902

18. Reyes-Godinez N, Ruiz-Lozano RE, Tamez-Tamez VE. Sudden vision loss associated with keratoconus: Acute corneal hydrops. Vis J Emerg Med. 2021;25:101109. doi:10.1016/j.visj.2021.101109

19. Kreps EO, Claerhout I, Koppen C. The Outcome of Scleral Lens Fitting for Keratoconus With Resolved Corneal Hydrops. Cornea. 2019;38(7):855-8. doi: 10.1097/ICO.0000000000001946 pmid: 31170103

20. Rathi VM, Dumpati S, Mandathara PS, Taneja MM, Sangwan VS. Scleral contact lenses in the management of pellucid marginal degeneration. Cont Lens Anterior Eye. 2016;39(3):217-20. doi: 10.1016/j.clae.2015.11.005 pmid: 26669275

21. Rathi VM, Murthy SI, Bagga B, Taneja M, Chaurasia S, Sangwan VS. Keratoglobus: An experience at a tertiary eye care center in India. Indian J Ophthalmol. 2015;63(3):233-8. doi: 4738.156927-0301/10.4103 pmid: 25971168

22. Pedrotti E, Passilongo M, Fasolo A, Ficial S, Ferrari S, Marchini G. Refractive outcomes of penetrating keratoplasty and deep anterior lamellar keratoplasty in fellow eyes for keratoconus. Int Ophthalmol. 2017;37(4):911-9. doi: 10.1007/s0-0350-016-10792 pmid: 27638316

23. Barnett M, Lien V, Li JY, Durbin-Johnson B, Mannis MJ. Use of Scleral Lenses and Miniscleral Lenses After Penetrating Keratoplasty. Eye Contact Lens. 2016;42(3):185-9. doi: 10.1097/ICL.0000000000000163 pmid: 26214530

24. Severinsky B, Behrman S, Frucht-Pery J, Solomon A. Scleral contact lenses for visual rehabilitation after penetrating keratoplasty: long term outcomes. Cont Lens Anterior Eye. 2014;37(3):196-202. doi: 10.1016/j.clae.2013.11.001 pmid: 24300196

25. Mian SZ, Agranat JS, Jacobs DS. Prosthetic Replacement of the Ocular Surface Ecosystem (PROSE) Treatment for Complications After LASIK. Eye Contact Lens. 2016;42(6):371-3. doi: 10.1097/ICL.0000000000000303 pmid: 27466722

26. Montalt JC, Porcar E, Espana-Gregori E, Peris-Martinez C. Corneal Biomechanical Parameters With Corneoscleral Contact Lenses in 
Post-Laser In Situ Keratomileusis Eyes. Eye Contact Lens. 2018;44:S65-S9. doi: 10.1097/ICL.0000000000000420 pmid: 28945645

27. Barrera-Sanchez M, Hernandez-Camarena J, Ruiz-Lozano R, Perez-Saucedo J, Rodriguez-Garcia A. The Value of Conjunctival Immunohistochemistry Analysis for the Diagnosis of Ocular Mucous Membrane Pemphigoid. Int J Ophthalmic Pathol 9. 2020;9(3):2. doi: 10.37532/iopj. 2020.9(2).238

28. Gomez-Elizondo DE, Lopez-Martinez M, Ruiz-Lozano RE, Valdez-Garcia JE, Hernandez-Camarena JC. Corneal perforation associated with isolated ocular lichen planus: a case report. Eur J Ophthalmol. 2020;31(6):NP6-NP12. doi: 1120672120932089/10.1177 pmid: 32493062

29. Ruiz-Lozano RE, Hernandez-Camarena JC, Loya-Garcia D, Merayo-Lloves J, Rodriguez-Garcia A. The molecular basis of neurotrophic keratopathy: Diagnostic and therapeutic implications. A review. Ocul Surf. 2020;19:224-40. doi: 10.1016/j. jtos.2020.09.007 pmid: 33022412

30. Parra AS, Roth BM, Nguyen TM, Wang L, Pflugfelder SC, Al-Mohtaseb Z. Assessment of the Prosthetic Replacement of Ocular Surface Ecosystem (PROSE) scleral lens on visual acuity for corneal irregularity and ocular surface disease. Ocul Surf. 2018;16(2):254-8. doi: 10.1016/j.jtos.2018.01.003 pmid: 29425812

31. Papakostas TD, Le HG, Chodosh J, Jacobs DS. Prosthetic replacement of the ocular surface ecosystem as treatment for ocular surface disease in patients with a history of Stevens-Johnson syndrome/toxic epidermal necrolysis. Ophthalmology. 2015;122(2):248-53. doi: 10.1016/j.ophtha.2014.08.015 pmid: 25282251

32. Wang Y, Rao R, Jacobs DS, Saeed HN. Prosthetic Replacement of the Ocular Surface Ecosystem Treatment for Ocular Surface Disease in Pediatric Patients With Stevens-Johnson Syndrome. Am J Ophthalmol. 2019;201:1-8. doi: 10.1016/j.ajo.2019.01.006 pmid: 30664843

33. Shikari H, Antin JH, Dana R. Ocular graft-versus-host disease: a review. Surv Ophthalmol. 2013;58(3):233-51. doi: 10.1016/j. survophthal.2012.08.004 pmid: 23541042

34. Magro L, Gauthier J, Richet M, Robin M, Nguyen S, Suarez F, et al. Scleral lenses for severe chronic GvHD-related keratoconjunctivitis sicca: a retrospective study by the SFGM-TC. Bone Marrow Transplant. 2017;52(6):878-82. doi: 10.1038/bmt.2017.9 pmid: 28218751

35. Rosenthal P, Croteau A. Fluid-ventilated, gas-permeable scleral contact lens is an effective option for managing severe ocular surface disease and many corneal disorders that would otherwise require penetrating keratoplasty. Eye Contact Lens. 2005;31(3):130-4. doi: 01/10.1097.icl.0000152492.98553.8d pmid: 15894881

36. Agranat JS, Kitos NR, Jacobs DS. Prosthetic replacement of the ocular surface ecosystem: impact at 5 years. Br J Ophthalmol. 2016;100(9):1171-5. doi: 10.1136/bjophthalmol-307483-2015 pmid: 26644423

37. Grey F, Carley F, Biswas S, Tromans C. Scleral contact lens management of bilateral exposure and neurotrophic keratopathy. Cont Lens Anterior Eye. 2012;35(6):288-91. doi: 10.1016/j.clae.2012.07.009 pmid: 22902053

38. Gervasio KA, Godfrey KJ, Marlow ED, Lee MN, Lelli GJ, Jr. Prosthetic Replacement of the Ocular Surface Ecosystem (PROSE) Versus Standard of Care for Postsurgical Lagophthalmos and Exposure Keratopathy: Trends in Visual Outcomes. Ophthalmic Plast Reconstr Surg. 2019;35(3):281-5. doi: 10.1097/IOP.0000000000001233 pmid: 30289823

39. Chahal JS, Heur M, Chiu GB. Prosthetic Replacement of the Ocular Surface Ecosystem Scleral Lens Therapy for Exposure Keratopathy. Eye Contact Lens. 2017;43(4):240-4 . doi: 10.1097/ICL.0000000000000265 pmid: 27171131

40. Lim P, Ridges R, Jacobs DS, Rosenthal P. Treatment of persistent corneal epithelial defect with overnight wear of a prosthetic device for the ocular surface. Am J Ophthalmol. 2013;156(6):1095-101. doi: 10.1016/j.ajo.2013.06.006 pmid: 24075432

41. Zimmerman AB, Marks A. Microbial keratitis secondary to unintended poor compliance with scleral gas-permeable contact lenses. Eye Contact Lens. 2014;40(1):e1-4. doi: 10.1097/ICL.0b013e318273420f pmid: 23392303

42. Ruiz-Lozano RE, Hernandez-Camarena JC, Valdez-Garcia JE, Roman-Zamudio M, Herrera-Rodriguez MI, Andrade-Carrillo D, et al. Ocular involvement and complications of lichen planus, lichen planus pigmentosus, and lichen planopilaris: A comprehensive review. Dermatol Ther. 2021. doi: 10.1111/dth.15137 pmid: 34541780

43. Vazirani J, Nair D, Shanbhag S, Wurity S, Ranjan A, Sangwan V. Limbal Stem Cell Deficiency-Demography and Underlying Causes. Am J Ophthalmol. 2018;188:99-103. doi: 10.1016/j.ajo.2018.01.020 pmid: 29378178

44. Vazirani J, Donthineni PR, Goel S, Sane SS, Mahuvakar S, Narang P, et al. Chronic cicatrizing conjunctivitis: A review of the differential diagnosis and an algorithmic approach to management. Indian J Ophthalmol. 2020;68(11):2349-55. doi: 10.4103/ijo. IJO_20_604 pmid: 33120615

45. Schornack MM, Baratz KH. Ocular cicatricial pemphigoid: the role of scleral lenses in disease management. Cornea. 2009;28(10):1170-2. doi: 10.1097/ICO.0b013e318199fa56 pmid: 19770718

46. Deng SX, Kruse F, Gomes JAP, Chan CC, Daya S, Dana R, et al. Global Consensus on the Management of Limbal Stem Cell Deficiency. Cornea. 2020;39(10):1291-302. doi: 10.1097/ICO.0000000000002358 pmid: 32639314

47. Rosenthal P, Cotter JM, Baum J. Treatment of persistent corneal epithelial defect with extended wear of a fluid-ventilated gaspermeable scleral contact lens. Am J Ophthalmol. 2000;130(1):33-41. doi: 10.1016/s2-00379(00)9394-0002 pmid: 11004257

48. Villarreal-Gonzalez AJ, Jocelyn Rivera-Alvarado I, Rodriguez-Gutierrez LA, Rodriguez-Garcia A. Analysis of ocular surface damage and visual impact in patients with primary and secondary Sjogren syndrome. Rheumatol Int. 2020;40(8):1249-57. doi: 10.1007/ s7-04568-020-00296 pmid: 32242261

49. Riega-Torres JCL, Trevino-Castro MA, Hernandez-Galarza IJ, Garza-Martinez MJ, Vera-Pineda R, Cardenas-de la Garza JA, et al. Cognitive dysfunction in Sjogren>s syndrome using the Montreal Cognitive Assessment Questionnaire and the Automated Neuropsychological Assessment Metrics: A cross-sectional study. Int J Rheum Dis. 2020;23(8):1019-23. doi: -1756/10.1111 185X.13889 pmid: 32608054

50. La Porta Weber S, Becco de Souza R, Gomes JAP, Hofling-Lima AL. The Use of the Esclera Scleral Contact Lens in the Treatment of Moderate to Severe Dry Eye Disease. Am J Ophthalmol. 2016;163:167-73 e1. doi: 10.1016/j.ajo.2015.11.034 pmid: 26701271

51. Weber SP, Hazarbassanov RM, Nasare A, Gomes JAP, Hofling-Lima AL. Conjunctival impression cytology evaluation of patients with dry eye disease using scleral contact lenses. Cont Lens Anterior Eye.2017;40(3):151-6 . doi: 10.1016/j.clae.2016.12.008 pmid: 28089075 
52. Moshirfar M, Bennett P, Ronquillo Y. Corneal Dystrophy. 2021. In: StatPearls [Internet]. Treasure Island (FL): StatPearls Publishing; 2022 Jan. pmid: 32491788

53. Barrera-Sanchez M, Hernandez-Camarena JC, Ruiz-Lozano RE, Valdez-Garcia JE, Rodriguez-Garcia A. Demographic profile and clinical course of Fuchs endothelial corneal dystrophy in Mexican patients. Int Ophthalmol. 2021. doi: 10.1007/s0-02117-021-10792 pmid: 34743257

54. Alipur F, Hosseini SS. Visual management of aphakia with concomitant severe corneal irregularity by mini-scleral design contact lenses. J Curr Ophthalmol. 2016;28(1):27-31. doi: 10.1016/j.joco.2016.01.004 pmid: 27239599

55. Gill FR (2010). 'Turning the tide for gas permeable contact lenses (Thesis)'. UMI Number: U585369. PhD dissertation, School of Optometry \& Vision Sciences, Cardiff University (United Kingdom). Available at: https://www.proquest.com/openview/f51622d4b 769214463851c9106d1/33932?pq-origsite=gscholar\&cbl=2026366 (Accessed: November 01, 2021)

56. Pullum KW, Whiting MA, Buckley RJ. Scleral contact lenses: the expanding role. Cornea. 2005;24(3):269-77. doi: 01/10.1097. ico.0000148311.94180.6b pmid: 15778597

57. Mutilab HA, Sharanjeet K, Keu LK, Choo PF. Special tinted contact lens on colour-defects. Clin Ter. 2012;163(3):199-204 pmid: 22964691

58. Kanemoto M, Toshida H, Takahiro I, Murakami A. Prosthetic soft contact lenses in Japan. Eye Contact Lens. 2007;33:300-3. doi: 10.1097/ICL.0b013e3180319ce9 pmid: 17993825

59. Katsoulos K, Rallatos GL, Mavrikakis I. Scleral contact lenses for the management of complicated ptosis. Orbit. 2018;37(3):201-7. doi: 01676830.2017.1383475/10.1080 pmid: 29053041

60. Pecego M, Barnett M, Mannis MJ, Durbin-Johnson B. Jupiter Scleral Lenses: the UC Davis Eye Center experience. Eye Contact Lens. 2012;38(3):179-82. doi: 10.1097/ICL.0b013e31824daa5e pmid: 22543730

61. Romero-Jiménez M, Flores-Rodríguez P. Utility of a semi-scleral contact lens design in the management of the irregular cornea. Cont Lens Anterior Eye. 2013;36(3):146-50. doi: 10.1016/j.clae.2012.12.006 pmid: 23291263

62. Rathi VM, Mandathara PS, Vaddavalli PK, Srikanth D, Sangwan VS. Fluid filled scleral contact lens in pediatric patients: challenges and outcome. Cont Lens Anterior Eye. 2012;35(4):189-92. doi: 10.1016/j.clae.2012.03.001 pmid: 22459875

63. Vincent SJ, Alonso-Caneiro D, Collins MJ, Beanland A, Lam L, Lim CC, et al. Hypoxic Corneal Changes following Eight Hours of Scleral Contact Lens Wear. Optom Vis Sci. 2016;93(3):293-9. doi: 10.1097/OPX.0000000000000803 pmid: 26760584

64. Kumar M, Shetty R, Khamar P, Vincent SJ. Scleral Lens-Induced Corneal Edema after Penetrating Keratoplasty. Optom Vis Sci. 2020;97(9):697-702. doi: 10.1097/OPX.0000000000001571 pmid: 32932397

65. Carnt N, Jalbert I, Stretton S, Naduvilath T, Papas E. Solution toxicity in soft contact lens daily wear is associated with corneal inflammation. Optom Vis Sci. 2007;84(4):309-15. doi: 10.1097/OPX.0b013e318046551b pmid: 17435514

66. Stapleton F, Bakkar M, Carnt N, Chalmers R, Vijay AK, Marasini S, et al. CLEAR - Contact lens complications. Cont Lens Anterior Eye. 2021;44(2):330-67. doi: 10.1016/j.clae.2021.02.010 pmid: 33775382

67. Walker MK, Bergmanson JP, Miller WL, Marsack JD, Johnson LA. Complications and fitting challenges associated with scleral contact lenses: A review. Cont Lens Anterior Eye. 2016;39(2):88-96. doi: 10.1016/j.clae.2015.08.003 pmid: 26341076

68. Fadel D, Toabe M. Scleral lens hygiene and care. Journal of Contact lens Research and Science. 2018;2(1):e30-7. doi: 10.22374/jclrs. v2i1.20

69. Visser ES, Visser R, van Lier HJ, Otten HM. Modern scleral lenses part II: patient satisfaction. Eye Contact Lens. 2007;33(1):21-5. doi: 01/10.1097.icl.0000228964.74647.25 pmid: 17224675

70. Barnett M, Johns L. Sceral lens Handling. Contemporary Scleral Lenses: Theory and Application Sharjah, UAE: Bentham Science Publishers. 70-2017:346. Link

71. Harthan J, Nau CB, Barr J, Nau A, Shorter E, Chimato NT, et al. Scleral Lens Prescription and Management Practices: The SCOPE Study. Eye Contact Lens. 2018;44 Suppl 1:S228-S32. doi: 10.1097/ICL.0000000000000387 pmid: 28394876

72. Tappin MJ, Pullum KW, Buckley RJ. Scleral contact lenses for overnight wear in the management of ocular surface disorders. Eye (Lond). 2001;15(Pt 2):168-72. doi: 10.1038/eye.2001.54 pmid: 11339584

73. Zhu B, Liu Y, Lin L, Huang X, Zhang Y, Zheng J, et al. Characteristics of Infectious Keratitis in Bandage Contact Lens Wear Patients. Eye Contact Lens. 2019;45(6):356-9. doi: 10.1097/ICL.0000000000000593 pmid: 31453822 